\title{
PROBABILITY AND QUANTA: WHY BACK TO NELSON?
}

\author{
PIOTR GARBACZEWSKI \\ Institute of Theoretical Physics, University of Wroctaw \\ pl. M. Borna 9, 50-204 Wroctaw, Poland \\ E-mail:pgar@ift.uni.wroc.pl
}

\begin{abstract}
We establish circumstances under which the dispersion of passive contaminants in a forced flow can be consistently interpreted as a Markovian diffusion process.

1. Introduction. Probabilistic concepts are ubiquitous in diverse areas of theoretical physics, in particular in connection with probabilistic models for various partial differential equations and their solutions. We shall name the common examples: Boltzmann, Navier-Stokes, Burgers, Euler, Fokker-Planck, Kramers, Schrödinger equation. The latter one, in conjunction with the Born statistical interpretation postulate, was the subject of an extensive research by E. Nelson, [11], and quite recently (while from a slightly different perspective) by K. L. Chung and Z. Zhao, [2]. A number of ideas due to Nelson, that were originally suited to analyze the stochastic counterpart of the Schrödinger picture quantum dynamics, gave an inspiration to a novel unifying framework of the so-called Schrödinger boundary data and stochastic interpolation problem, $[15,5,7,8,4]$. That sets a conceptual and formal (stochastic analysis methods) basis for a surprisingly rich group of topics being potentially of interest to both mathematicians and physicists. They range from stochastic processes of the nonequlibrium statistical physics, through classical dynamics of complex systems (chaos in terms of densities) to quantum theory. On the other hand they involve random processes which are compatible with the infinitely divisible probability laws and their more general (perturbed) versions, hence go beyond the diffusion process setting. A sample of arguments taken from this line of active research is presented below.
\end{abstract}

2. The Schrödinger boundary data problem and Markovian interpolating dynamics. According to pedestrian intuitions, one normally expects that any "reasonable" kind of time developement (dynamics, be it deterministic or random), which is

1991 Mathematics Subject Classification: Primary 60H30; Secondary 60J60.

The paper is in final form and no version of it will be published elsewhere. 
analyzable in terms of probability, under suitable mathematical restrictions may give rise to a well defined stochastic process. An inverse operation of deducing the detailed (possibly individual, microscopic) dynamics, which is either compatible with a given probability measure (we shall be mostly interested in those admitting densities) or is compatible with its time evolution, does not have a unique solution. However, the level of ambiguities can be substantially reduced, if we invoke the so-called Schrödinger problem of reconstructing the microscopic dynamics from the given input-output statistics data or from the a priori known (evolving in time) probability density. That gives rise to a particular class of solutions (most likely interpolations), in terms of Markov stochastic processes, and diffusion processes in particular, [5, 4].

In its original formulation, due to Schrödinger, $[14,15]$, one seeks the answer to the following question: given two strictly positive (usually on an open space-interval) boundary probability densities $\rho_{0}(\vec{x}), \rho_{T}(\vec{x})$ for a process with the time of duration $T \geq 0$. Can we uniquely identify the stochastic process interpolating between them? The answer is known to be affirmative and, in particular, one can ultimately single out a unique Markovian diffusion process which is specified by solving the (Schrödinger) boundary data problem:

$$
\begin{gathered}
m_{T}(A, B)=\int_{A} d^{3} x \int_{B} d^{3} y m_{T}(\vec{x}, \vec{y}) \\
\int d^{3} y m_{T}(\vec{x}, \vec{y})=\rho_{0}(\vec{x}) \\
\int d^{3} x m_{T}(\vec{x}, \vec{y})=\rho_{T}(y)
\end{gathered}
$$

where the joint probability distribution has a bi-variate density in the specific factorised form

$$
m_{T}(\vec{x}, \vec{y})=u_{0}(\vec{x}) k(x, 0, y, T) v_{T}(\vec{y})
$$

and the two unknown functions $u_{0}(\vec{x}), v_{T}(\vec{y})$ come out as solutions of the same sign of the integral identities (1). Provided we have at our disposal a continuous bounded strictly positive (ways to relax this assumption were discussed in Ref. [6]) function $k(\vec{x}, s, \vec{y}, t), 0 \leq s<t \leq T$, which for our purposes (an obvious way to secure the Markov property) is chosen to be represented by familiar Feynman-Kac integral kernels of contractive dynamical semigroup operators:

$$
k(\vec{y}, s, \vec{x}, t)=\int \exp \left[-\int_{s}^{t} c(\vec{\omega}(\tau), \tau) d \tau\right] d \mu_{(\vec{x}, t)}^{(\vec{y}, s)}(\omega) .
$$

The pertinent (interpolating) Markovian process can be determined by checking (this imposes limitations on the admissible function $c$ ) whether the Feynman-Kac kernel is positive and continuous in the open space-time area of interest (then, additional limitations on the path measure need to be introduced, [1]), and whether it gives rise to positive solutions (it is desirable to have them bounded, $[3,2]$ ) of the adjoint pair of generalised heat equations:

$$
\begin{gathered}
\partial_{t} u(\vec{x}, t)=\nu \triangle u(\vec{x}, t)-c(\vec{x}, t) u(\vec{x}, t) \\
\partial_{t} v(\vec{x}, t)=-\nu \triangle v(\vec{x}, t)+c(\vec{x}, t) v(\vec{x}, t) .
\end{gathered}
$$


Here, a function $c(\vec{x}, t)$ is restricted only by the positivity and continuity demand for the kernel $(3)$, see e.g. $[5,2,3]$. In the above, $d \mu_{(\vec{x}, t)}^{(\vec{y}, s)}(\omega)$ is the conditional Wiener measure over sample paths of the standard Brownian motion.

Solutions of (4) upon suitable normalisation give rise to the Markovian diffusion process with the factorised probability density $\rho(\vec{x}, t)=u(\vec{x}, t) v(\vec{x}, t)$ which, while evolving in time, interpolates between the boundary density data $\rho(\vec{x}, 0)$ and $\rho(\vec{x}, T)$. The interpolation admits an Itô realisation with the respective forward and backward drifts defined as follows:

$$
\begin{aligned}
\vec{b}(\vec{x}, t) & =2 \nu \frac{\nabla v(\vec{x}, t)}{v(\vec{x}, t)} \\
\vec{b}_{*}(\vec{x}, t) & =-2 \nu \frac{\nabla u(\vec{x}, t)}{u(\vec{x}, t)}
\end{aligned}
$$

in the prescribed time interval $[0, T]$.

The related transport equations for the densities easily follow. For the forward interpolation, the familiar Fokker-Planck (second Kolmogorov) equation holds true:

$$
\partial_{t} \rho(\vec{x}, t)=\nu \triangle \rho(\vec{x}, t)-\nabla[\vec{b}(\vec{x}, t) \rho(\vec{x}, t)]
$$

while for the backward interpolation we have:

$$
\partial_{t} \rho(\vec{x}, t)=-\nu \triangle \rho(\vec{x}, t)-\nabla\left[\vec{b}_{*}(\vec{x}, t) \rho(\vec{x}, t)\right] .
$$

By (5) the drifts are gradient fields, $\operatorname{curl} \vec{b}=0$. As a consequence, those that are allowed by any prescribed choice of the function $c(\vec{x}, t)$ must fulfill the compatibility condition

$$
c(\vec{x}, t)=\partial_{t} \Phi+\frac{1}{2}\left(\frac{b^{2}}{2 \nu}+\nabla b\right)
$$

which establishes the Girsanov-type connection of the forward drift $\vec{b}(\vec{x}, t)=2 \nu \nabla \Phi(\vec{x}, t)$ with the Feynman-Kac, cf. [1], potential (at this point we follow the physical terminology) $c(\vec{x}, t)$. In the considered Schrödinger's interpolation framework, the forward and backward drift fields are connected by the identity $\vec{b}_{*}=\vec{b}-2 \nu \nabla \ln \rho$.

One of the distinctive features of Markovian diffusion processes with the positive density $\rho(\vec{x}, t)$ is that, given the transition probability density of the (forward) process, the notion of the backward transition probability density $p_{*}(\vec{y}, s, \vec{x}, t)$ can be consistently introduced on each finite time interval, say $0 \leq s<t \leq T$ :

$$
\rho(\vec{x}, t) p_{*}(\vec{y}, s, \vec{x}, t)=p(\vec{y}, s, \vec{x}, t) \rho(\vec{y}, s)
$$

so that $\int \rho(\vec{y}, s) p(\vec{y}, s, \vec{x}, t) d^{3} y=\rho(\vec{x}, t)$ and $\rho(\vec{y}, s)=\int p_{*}(\vec{y}, s, \vec{x}, t) \rho(\vec{x}, t) d^{3} x$.

The transport (density evolution) equations (6) and (7) refer to processes running in opposite directions in a fixed, common for both, time-duration period. The forward one, (6), executes an interpolation from the Borel set $A$ to $B$, while the backward one, (7), executes an interpolation from $B$ to $A$, compare e.g. the defining identities (1).

The knowledge of the Feynman-Kac kernel (3) implies that the transition probability density of the forward process reads:

$$
p(\vec{y}, s, \vec{x}, t)=k(\vec{y}, s, \vec{x}, t) \frac{v(\vec{x}, t)}{v(\vec{y}, s)},
$$


while the corresponding (derivable from $(10)$, since $\rho(\vec{x}, t)$ is given) transition probability density of the backward process has the form:

$$
p_{*}(\vec{y}, s, \vec{x}, t)=k(\vec{y}, s, \vec{x}, t) \frac{u(\vec{y}, s)}{u(\vec{x}, t)} .
$$

Obviously, $[5,15]$, in the time interval $0 \leq s<t \leq T$ there holds:

$$
\begin{gathered}
u(\vec{x}, t)=\int u_{0}(\vec{y}) k(\vec{y}, s, \vec{x}, t) d^{3} y \\
v(\vec{y}, s)=\int k(\vec{y}, s, \vec{x}, T) v_{T}(\vec{x}) d^{3} x .
\end{gathered}
$$

Consequently, the system (4) fully determines the underlying random motions, forward and backward, respectively.

3. Diffusion process in a forced flow. Whenever one tries to analyze random perturbations that are either superimposed upon or intrinsic to a driving deterministic motion, quite typically a configuration space equation $\dot{\vec{x}}=\vec{v}(\vec{x}, t)$ is invoked, which is next replaced by a formal infinitesimal representation of an Itô diffusion process $d \vec{X}(t)=$ $\vec{b}(\vec{X}(t), t) d t+\sqrt{2 \nu} d \vec{W}(t)$. Here, $\vec{W}(t)$ stands for the normalised Wiener noise, and $\nu$ for a diffusion constant, cf. (4)-(12).

The dynamical meaning of $\vec{b}(\vec{x}, t)$ relies on a specific diffusion input and its possible phase-space (e.g. Langevin) implementation, that entail a detailed functional relationship of $\vec{v}(\vec{x}, t)$ and $\vec{b}(\vec{x}, t)$, and justify such notions like: diffusion in an external force field, diffusion along, against or across the deterministic flow, $[9,13]$. The pertinent mathematical formalism corroborates both the Brownian motion of a single particle and the diffusive transport of neutrally buoyant components in flows of the hydrodynamic type.

Clearly, in random media that are statistically at rest, diffusion of single tracers or dispersion of pollutants are well described by the Fickian outcome of the molecular agitation, also in the presence of external force fields (then in terms of Smoluchowski diffusions). On the other hand, it is of fundamental importance to understand how statistically relevant flows in a random medium (fluid, as example) affect dispersion. In the context of fluids, we might refer to diffusion enhancement due to turbulence, behaviour of Brownian particles in shear flows, but also to general effects of the external forcing (various forms of deterministic or random "stirring" of the random medium) exerted upon gradient or non-gradient, compressible and incompressible flows, and carried by them passive constituents.

Except for suitable continuity and growth restrictions, necessary to guarantee the existence of the process $\vec{X}(t)$ governed by the Itô stochastic differential equation, the choice of the driving velocity field $\vec{v}(\vec{x}, t)$ and hence of the related drift $\vec{b}(\vec{x}, t)$ is normally regarded to be arbitrary.

However, the situation looks otherwise, if we are interested in a diffusion of passive tracers in the a priori given flow whose velocity field is a solution of the nonlinear partial differential equation, be it Euler, Navier-Stokes, Burgers or the like. An implicit assumption, that tracers passively buoyant in a fluid have a negligible effect on the flow, looks acceptable (basically, if the concentration of a passive component in a flow is small). Then, one is tempted to view directly the fluid velocity field $\vec{v}(\vec{x}, t)$ as the forward drift 
$\vec{b}(\vec{x}, t)$ of the process, with the contaminant being diffusively dispersed along the streamlines. But, in general, the assumed nonlinear evolution rule for $\vec{v}(\vec{x}, t)$ must be checked against the dynamics that is allowed to govern the space-time dependence of the forward drift field $\vec{b}(\vec{x}, t)$, [11], which is not at all arbitrary. The latter is ruled by standard consistency conditions that are respected by any Markovian diffusion process, and additionally by the rules of the forward and backward Itô calculus, [9, 11].

This particular issue we have analyzed before in the context of Burgers flows, [8], where the Burgers velocity field was found to be inappropriate to stand for the forward drift of a Markovian diffusion process. Actually, the backward drift was a correct identification. Then, the forced Burgers dynamics (at this point we shall slightly adjust the previous convention by defining $\Omega(\vec{x}, t)=2 \nu c(\vec{x}, t)$, see Eq. $(3))$

$$
\partial_{t} \vec{v}_{B}+\left(\vec{v}_{B} \cdot \vec{\nabla}\right) \vec{v}_{B}=\nu \triangle \vec{v}_{B}+\vec{\nabla} \Omega
$$

and the diffusion-convection equation

$$
\partial_{t} \sigma+\left(\vec{v}_{B} \cdot \vec{\nabla}\right) \sigma=\nu \triangle \sigma
$$

for the concentration $\sigma(\vec{x}, t)$ of a passive component in a flow, in case of gradient velocity fields, were proved to be compatible with the Markovian diffusion process input.

According to Ref. [8], in that case the dynamics of concentration results from the stochastic diffusion process whose density $\rho(\vec{x}, t)$ evolves according to $(7)$

$$
\partial_{t} \rho=-\nu \triangle \rho-\vec{\nabla} \cdot\left(\vec{v}_{B} \rho\right)
$$

or equivalently:

$$
\begin{aligned}
\partial_{t} \rho & =\nu \Delta \rho-\vec{\nabla} \cdot(\vec{b} \rho), \\
\vec{b} & \doteq \vec{v}_{B}+2 \nu \vec{\nabla} \ln \rho .
\end{aligned}
$$

In case of conservative forcing, the forward drift solves an evolution equation:

$$
\partial_{t} \vec{b}+(\vec{b} \cdot \vec{\nabla}) \vec{b}=-\nu \triangle \vec{b}+\vec{\nabla} \Omega .
$$

For drifts that are gradient fields, the function $\Omega$, whatever its functional form is, must allow for a representation formula, which is reminiscent of the Cameron-Martin-Girsanov transformation, (8) i.e. $\Omega(\vec{x}, t)=2 \nu\left[\partial_{t} \Phi+\frac{1}{2}\left(\frac{\vec{b}^{2}}{2 \nu}+\vec{\nabla} \cdot \vec{b}\right)\right]$, where $\vec{b}(\vec{x}, t)=\vec{\nabla} \Phi(\vec{x}, t)$.

For the existence of the Markovian diffusion process with the forward drift $\vec{b}(\vec{x}, t)$, we must resort to potentials (physicist's notion) $\Omega(\vec{x}, t)$ that are not completely arbitrary functions. Technically, $[5,2,3]$, the minimal requirement is that the potential is bounded from below. This restriction will have profound consequences for our further discussion of a diffusion process in a flow.

If we set $\rho=\rho_{1}+\rho_{2}$, and demand that $\rho_{1} \neq \rho$ solves the Fokker-Planck equation with the very same $\operatorname{drift} \vec{b}(\vec{x}, t)$ as $\rho$ does, then as a necessary consequence of the general formalism, $[8,5]$, the concentration

$$
\sigma(\vec{x}, t)=\frac{\rho_{1}(\vec{x}, t)}{\rho(\vec{x}, t)}
$$

solves an associated diffusion-convection equation (14). Here, the flow velocity $\vec{v}_{B}(\vec{x}, t)$ coincides with the backward drift $\vec{b}_{*} \doteq \vec{v}_{B}$ of the generic diffusion process with the density 
$\rho(\vec{x}, t)$ and reads: $\vec{v}_{B}=\vec{b}-2 \nu \vec{\nabla} \ln \rho$. Obviously, the forced Burgers equation (13) is identically satisfied.

We should clearly discriminate between forces whose effect is a "stirring" of the random medium and those acting selectively on diffusing particles, with a negligible effect on the medium itself. For example, the traditional Smoluchowski diffusion processes in conservative force fields are considered in random media that are statistically at rest. Following the standard (phase-space, Langevin) methodology, let us set $\vec{b}(\vec{x})=\frac{1}{\beta} \vec{K}(\vec{x})$, where $\beta$ is a (large) friction coefficient and $\vec{K}$ represents an external Newtonian force per unit of mass ( e.g. an acceleration) that is of gradient from, $\vec{K}=-\vec{\nabla} U$. Then, the effective potential $\Omega$ reads: $\Omega=\frac{\vec{K}^{2}}{2 \beta^{2}}+\frac{\nu}{\beta} \vec{\nabla} \cdot \vec{K}$ and the only distinction between the attractive or repulsive cases can be read out from the term $\vec{\nabla} \cdot \vec{K}$.

4. Back to Nelson. In the above Eq. (13), by formally changing the sign of $\Omega$, we would arrive at the attractive variant of the problem that is however incompatible with the diffusion process scenario in view of the unboundedness of $-\Omega$ from below. This is the major point of our discussion: we may get in serious trouble with the Markovian diffusion input in case of general external "stirring" forces. Hence, we must specify an admissible class of perturbations which, while modifying the flow dynamics, would nonetheless generate a consistent diffusion-in-a-flow transport of passive tracers.

Should we a priori exclude the attractive variants of the potential $\Omega$ ? Can we save the situation by incorporating, hitherto not considered, "pressure" term effects as suggested by the general form of the compressible Euler (here $\vec{F}=-\vec{\nabla} V$ stands for external volume forces and $\rho$ for the fluid density that itself undergoes a stochastic diffusion process):

$$
\partial_{t} \vec{v}_{E}+\left(\vec{v}_{E} \cdot \vec{\nabla}\right) \vec{v}_{E}=\vec{F}-\frac{1}{\rho} \vec{\nabla} P
$$

or the incompressible, [10], Navier-Stokes equation:

$$
\partial_{t} \vec{v}_{N S}+\left(\vec{v}_{N S} \cdot \vec{\nabla}\right) \vec{v}_{N S}=\frac{\nu}{\rho} \triangle \vec{v}_{N S}+\vec{F}-\frac{1}{\rho} \vec{\nabla} P
$$

both to be compared with the equations (13) and (17), that set dynamical constraints for respectively backward and forward drifts of a Markovian diffusion process?

Notice that the acceleration term $\vec{F}$ in equations (19) and (20) normally is regarded as arbitrary, while the corresponding term $\vec{\nabla} \Omega$ in (13) and (17) involves a bounded from below function $\Omega(\vec{x}, t)$.

Since, in case of gradient velocity fields, the dissipation term in the incompressible Navier-Stokes equation (20) identically vanishes, we should concentrate on analyzing the possible "forward drift of the Markovian process" meaning of the Euler flow with the velocity field $\vec{v}_{E},(19)$. At this point it is useful, at least on the formal grouds, to invoke the standard phase-space argument that is valid for a Markovian diffusion process taking place in a given flow $\vec{v}(\vec{x}, t)$ with as yet unspecified (flow) dynamics. We account for an explicit force exerted upon diffusing particles, while not necessarily directly affecting the driving flow itself. Namely, [11], let us set for infinitesimal increments of phase space random variables: 


$$
\begin{gathered}
d \vec{X}(t)=\vec{V}(t) d t \\
d \vec{V}(t)=\beta[\vec{v}(\vec{x}, t)-\vec{V}(t)] d t+\vec{K}(\vec{x}) d t+\beta \sqrt{2 \nu} d \vec{W}(t) .
\end{gathered}
$$

Following the leading idea of the Smoluchowski approximation, we assume that $\beta$ is large, and consider the process for times significantly exceeding $\beta^{-1}$. Then, an appropriate choice of the velocity field $\vec{v}(\vec{x}, t)$ (boundedness and growth restrictions are involved) may in principle guarrantee, [11], the convergence of the spatial part $\vec{X}(t)$ of the process (21) to the Itô diffusion process with infinitesimal increments:

$$
d \vec{X}(t)=\vec{v}(\vec{x}, t) d t+\sqrt{2 \nu} d \vec{W}(t) .
$$

However, one cannot blindly insert in the place of the forward drift $\vec{v}(\vec{x}, t)$ any of the previously considered bulk velocity fields, without going into apparent contradictions. Specifically, the equation (13) with $\vec{v}(\vec{x}, t) \leftrightarrow \vec{b}(\vec{x}, t)$ must be valid. By resorting to velocity fields $\vec{v}(\vec{x}, t)$ which obey $\triangle \vec{v}(\vec{x}, t)=0$, we may pass from (13) to an equation of the Euler form, (19), provided (8) holds true and then the right-hand-side of (13) involves effective potential $\Omega$ bounded from below.

An additional requirement is that

$$
\vec{F}-\frac{1}{\rho} \vec{\nabla} P \doteq \vec{\nabla} \Omega
$$

Clearly, for a constant pressure we are left with the dynamical constraint $\left(\vec{b} \leftrightarrow \vec{v}_{E}\right)$ :

$$
\partial_{t} \vec{b}+(\vec{b} \cdot \vec{\nabla}) \vec{b}=\vec{F}=\vec{\nabla} \Omega
$$

combining simultaneously the Eulerian fluid and the Markov diffusion process inputs, if and only if $\vec{F}$ is repulsive, e.g. $-V(\vec{x}, t)$ is bounded from below. Quite analogously, by setting $\vec{F}=\overrightarrow{0}$, we would get a constraint on the admissible pressure term, in view of:

$$
\partial_{t} \vec{b}+(\vec{b} \cdot \vec{\nabla}) \vec{b}=-\frac{1}{\rho} \vec{\nabla} P=\vec{\nabla} \Omega .
$$

Both, in cases (24), (25) the effective potential $\Omega$ must respect the functional dependence (on a forward drift and its potential) prescription (8). In addition, the FokkerPlanck equation with the forward drift $\vec{v}_{E}(\vec{x}, t) \doteq \vec{b}(\vec{x}, t)$ must be valid for the density $\rho(\vec{x}, t)$.

To our knowledge, in the literature there is known only one specific class of Markovian diffusion processes that would render the right-hand-side of Eq. (19) repulsive but nevertheless account for the troublesome Newtonian accelerations, e.g. those of the form $-\vec{\nabla} V$, with $+V$ bounded from below. Such processes have forward drifts that for each suitable function $V(\vec{x})$ bounded from below solve the nonlinear partial differential equation:

$$
\partial_{t} \vec{b}+(\vec{b} \cdot \vec{\nabla}) \vec{b}=-\nu \triangle \vec{b}+\vec{\nabla}(2 Q-V)
$$

with the compensating pressure term:

$$
\begin{gathered}
Q \doteq 2 \nu^{2} \frac{\triangle \rho^{1 / 2}}{\rho^{1 / 2}} \doteq \frac{1}{2} \vec{u}^{2}+\nu \vec{\nabla} \cdot \vec{u} \\
\vec{u}(\vec{x}, t)=\nu \vec{\nabla} \ln \rho(\vec{x}, t) .
\end{gathered}
$$


Their exhaustive discussion can be found in Refs. [11, 8, 5, 15], together with indications for their possible relevance as a stochastic counterpart of the Schrödinger picture quantum dynamics. Clearly, we have:

$$
\vec{F}=-\vec{\nabla} V, \vec{\nabla} 2 Q=-\frac{1}{\rho} \vec{\nabla} P
$$

where:

$$
P(\vec{x}, t)=-2 \nu^{2} \rho(\vec{x}, t) \triangle \ln \rho(\vec{x}, t) .
$$

Effectively, $P$ is here defined up to a time-dependent constant. Another admissible form of the pressure term reads (summation convention is implicit):

$$
\frac{1}{\rho} \vec{\nabla}_{k}\left[\rho\left(2 \nu^{2} \partial_{j} \partial_{k}\right) \ln \rho\right]=\vec{\nabla}_{j}(2 Q) .
$$

If we consider a subclass of processes for which the dissipation term identically vanishes (a number of examples is known)

$$
\triangle \vec{b}(\vec{x}, t)=0
$$

the equation (26) takes a conspicuous Euler form (19), $\vec{v}_{E} \leftrightarrow \vec{b}$.

Let us notice that (29), (30) provide a generalisation of the more familiar equation of state $P \sim \rho$, thermodynamically motivated and suited for ideal gases and fluids. For density fields for which $-\triangle \ln \rho \sim$ const, the standard relationship between the pressure and the density is recovered. For density fields obeying $-\triangle \ln \rho=0$, we are left with at most purely time dependent or a constant pressure. Pressure profiles may be highly complex for arbitrarily chosen initial density and/or the flow velocity fields.

To conclude the present discussion let us invoke Refs. [10, 12, 5] The problem of a diffusion process interpretation of various partial differential equations has been extended beyond the original parabolic equations setting, to nonlinear velocity field equations like the Burgers one, see e.g. [8]. On the other hand, the nonlinear Markov processes associated with the Boltzmann equation, in the hydrodynamic limit, are known to imply either an ordinary differential equation with the velocity field solving the Euler equation, or a diffusion process whose drift is a solution of the incompressible Navier-Stokes equation (without the curl $\vec{v}=0$ restriction), [12, 10]. The case of external forcing has never been satisfactorily solved.

Our reasoning went otherwise. We asked for the admissible space-time dependence of general velocity fields that are to play the rôle of forward drifts of Markovian diffusion processes. Our finding is that solutions of the compressible Euler equation are appropriate for the description of a non-deterministic (e.g. random and Markovian) evolution and belong to a class of Markovian diffusion processes orginally introduced by E. Nelson in his quest for a probabilistic counterpart of the quantum dynamics, [11, 5]. Our solution of the problem involves only the gradient velocity fields. However, a couple of issues concerning the curl $\vec{b} \neq 0$ velocity fields and their nonconservative forcing have been raised in Refs. [8], with an additional inspiration coming from Nelson's investigations. 


\section{References}

[1] Ph. Blanchard and P. Garbaczewski, Natural boundaries for the Smoluchowski equation and affiliated diffusion processes, Phys. Rev. E 49, (1994), 3815.

[2] K. L. Chung and Z. Zhao, From Brownian Motion to Schrödinger Equation, SpringerVerlag, Berlin, 1995.

[3] M. Freidlin, Functional Integration and Partial Differential Equations, Princeton University Press, Princeton, 1985.

[4] P. Garbaczewski, J. R. Klauder and R. Olkiewicz, Schrödinger problem, Lévy processes, and noise in relativistic quantum mechanics, Phys. Rev. E 51, (1995), 4114.

[5] P. Garbaczewski and R. Olkiewicz, Feynman-Kac kernels in Markovian representations of the Schrödinger interpolating dynamics, J. Math. Phys. 37, (1996), 730.

[6] P. Garbaczewski, Schrödinger's interpolation problem through Feynman-Kac kernels, Acta Phys. Polon. B 27, (1996), 617.

[7] P.Garbaczewski and G. Kondrat, Burgers velocity fields and dynamical transport processes, Phys. Rev. Lett. 77, (1996), 2608.

[8] P. Garbaczewski, G. Kondrat and R. Olkiewicz, Burgers flows as Markovian diffusion processes, Phys. Rev. E 55, (1997), 1401.

[9] W. Horsthemke and R. Lefever, Noise-Induced Transitions, Springer-Verlag, Berlin, 1984.

[10] C. Marchioro and M. Pulvirenti, Vortex methods in Two-Dimensional Fluid Dynamics, Lecture Notes in Physics 203, Springer-Verlag, Berlin, 1984.

[11] E. Nelson, Dynamical Theories of the Brownian Motion, Princeton University Press, Princeton, 1967.

[12] H. Spohn, Large Scale Dynamics of Interacting Particles, Springer-Verlag, Berlin, 1992.

[13] H. Risken, The Fokker-Planck Equation, Springer-Verlag, Berlin, 1989.

[14] E. Schrödinger, Relativistic electron, Ann. Inst. Henri Poincaré, 2, (1932), 269.

[15] J. C. Zambrini, Variational processes and stochastic versions of mechanics, J. Math. Phys. 27, (1986), 3207. 